\title{
CARCINOMA DE CÉLULAS FUSIFORMES DE ESÔFAGO: RELATO DE CASO E REVISÃO DA LITERATURA*
}

\author{
Ricardo Hideki Yanaga ${ }^{1}$, Jorge Alberto Ledesma ${ }^{2}$, Carolina Souza Althoff ${ }^{1}$, Maria Fernanda \\ Soares $^{3}$
}

\begin{abstract}
Resumo Lesões malignas do esôfago, excluindo-se carcinomas escamocelulares e adenocarcinomas, são bastante incomuns. Os autores relatam um caso de carcinoma de células fusiformes de esôfago. É um tumor maligno raro caracterizado por volumosa massa lobulada no terço médio do esôfago, que causa relativamente pequena obstrução apesar do seu volume. Em seguida é apresentada revisão da literatura sobre o assunto. Unitermos: Esôfago; Carcinoma de células fusiformes; Carcinossarcoma; Pseudo-sarcoma.
\end{abstract}

Abstract Spindle cell carcinoma of the esophagus: case report and review of the literature.

Malignant lesions of the esophagus, other than epidermoids and adenocarcinomas, are remarkably uncommon. The authors report a case of a patient with a spindle cell carcinoma of the esophagus. This is a rare malignant tumor characterized by a large bulky lobulated mass in the middle third of the esophagus that causes relatively little obstruction in spite of its volume. A review of literature is presented. Key words: Esophagus; Spindle cell carcinoma; Carcinosarcoma; Pseudosarcoma.

\section{INTRODUÇÃO}

Lesões malignas que acometem o esôfago, excluindo-se os carcinomas escamocelulares (CEC) e adenocarcinomas, são bastante incomuns. O carcinoma de células fusiformes (CCF) de esôfago é uma neoplasia maligna rara, caracterizada por ser constituída por mistura de células epiteliais (componente carcinomatoso) e fusiformes (componente sarcomatoso) ${ }^{(\mathbf{1 - 6})}$. Tais tumores também foram descritos no útero, vagina, pulmões, cavidade oral, laringe, tireóide e trato urinário ${ }^{(\mathbf{1 , 3})}$. Devido ao seu componente sarcomatoso, são caracterizados por grandes massas polipóides e lobuladas no terço médio do esôfago. Tipicamente expandem e dilatam a luz do esôfago, causando relativamente pequena obstrução, desproporcional ao tamanho tumoral $^{(\mathbf{1 , 2 , 4 )} \text {. }}$

Esta entidade foi conhecida em 1864 por Virchow ${ }^{(7)}$, com o termo de carcinossarcoma, e em 1903 Von Hansemann ${ }^{(8)}$

* Trabalho realizado no Serviço de Radiologia do Hospital de Clínicas da Universidade Federal do Paraná (UFPR), Curitiba, PR.

1. Médicos Residentes de Radiologia do Hospital de Clínicas da UFPR

2. Médico Radiologista do Hospital de Clínicas da UFPR.

3. Médica Residente de Anatomia Patológica do Hospital de Clínicas da UFPR.

Endereço para correspondência: Dr. Ricardo Hideki Yanaga. Avenida Presidente Affonso Camargo, 849, apto. 602B, Cristo Rei. Curitiba, PR, 80050-370. E-mail: ricyanaga@bol.com.br

Recebido para publicação em 22/7/2002. Aceito, após revisão, em 31/10/2002. descreveu dois casos, pela primeira vez encontrados no esôfago. Desde então, poucos casos têm sido relatados na literatura mundial.

O presente caso é descrito para chamar a atenção para esta lesão rara e discutir suas características clínicas, radiológicas e patológicas

\section{RELATO DO CASO}

Paciente do sexo masculino, 42 anos de idade, tabagista e ex-etilista, há três meses apresentando disfagia progressiva para sólidos, dor em queimação retroesternal e emagrecimento de $4 \mathrm{~kg}$. Ao exame físico não se observou nenhuma particularidade.

A seriografia demonstrou volumosa falha de enchimento polipóide, lobulada, localizado no terço médio do esôfago, expandindo e dilatando a luz, sem causar obstrução significativa à passagem do meio de contraste (Figura 1).

A tomografia computadorizada do tórax demonstrou aumento de volume e espessamento parietal do esôfago abaixo da carina, sem evidências de infiltração de estruturas adjacentes ou disseminação para o parênquima pulmonar (Figura 2).

A endoscopia digestiva alta mostrou presença de lesão vegetante, sólida, com superfície irregular e algumas ulcerações, fixada à parede posterior do esôfago, localizada a $32 \mathrm{~cm}$ da arcada dentária. A bióp- sia sugeriu diagnóstico citológico de carcinoma indiferenciado.

O paciente foi submetido a esofagectomia total, com anastomose gástrica cervical. Ao estudo histopatológico observouse lesão polipóide e ulcerada, medindo 6,5 $\times 2,7 \times 1,5 \mathrm{~cm}$, com margem tumoral de padrão expansivo, constituída de tecido finamente granular e fosco, de consistência firme e friável. Havia invasão até a adventícia, ausência de invasão vascular e perineural, ausência de metástases para linfonodos regionais dissecados e margens cirúrgicas livres. A microscopia evidenciou que a lesão era constituída de mistura de elementos carcinomatosos (células com áreas de queratinização concêntrica) e sarcomatosos (células fusocelulares com intensas atipias nucleares) (Figura 3). O estudo imuno-histoquímico demonstrou detecção positiva para células fusiformes e epitelióides, confirmando o diagnóstico de CEC pouco diferenciado variante de células fusiformes.

\section{DISCUSSÃO}

Tumores malignos polipóides epiteliais do esôfago contendo ambos os elementos - carcinomatosos e sarcomatosos — são raros, correspondendo a apenas $0,5 \%$ a $1,5 \%$ de todas as neoplasias esofágicas ${ }^{(2,3)}$. Termos utilizados para descrever essas lesões incluem carcinossarcoma, pseudo- 


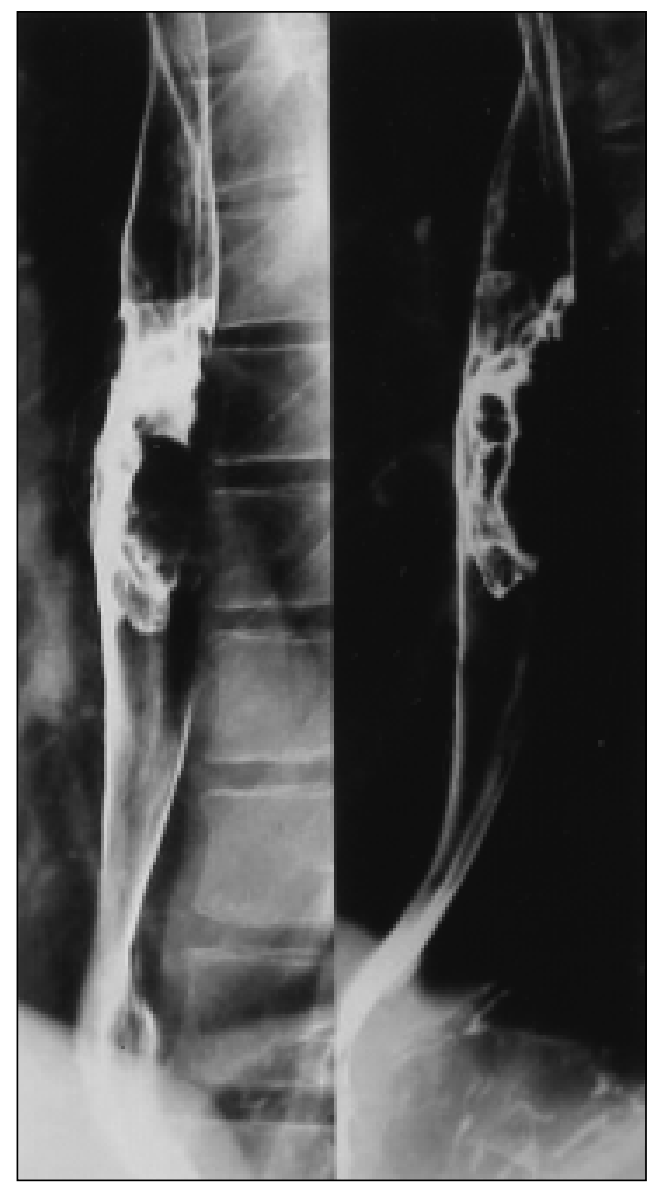

A

Figura 1. Seriografia. A: Massa polipóide, lobulada e expansiva localizada no terço médio do esôfago. B: Obstrução à passagem do meio de contraste proporcionalmente menor ao tamanho do tumor.

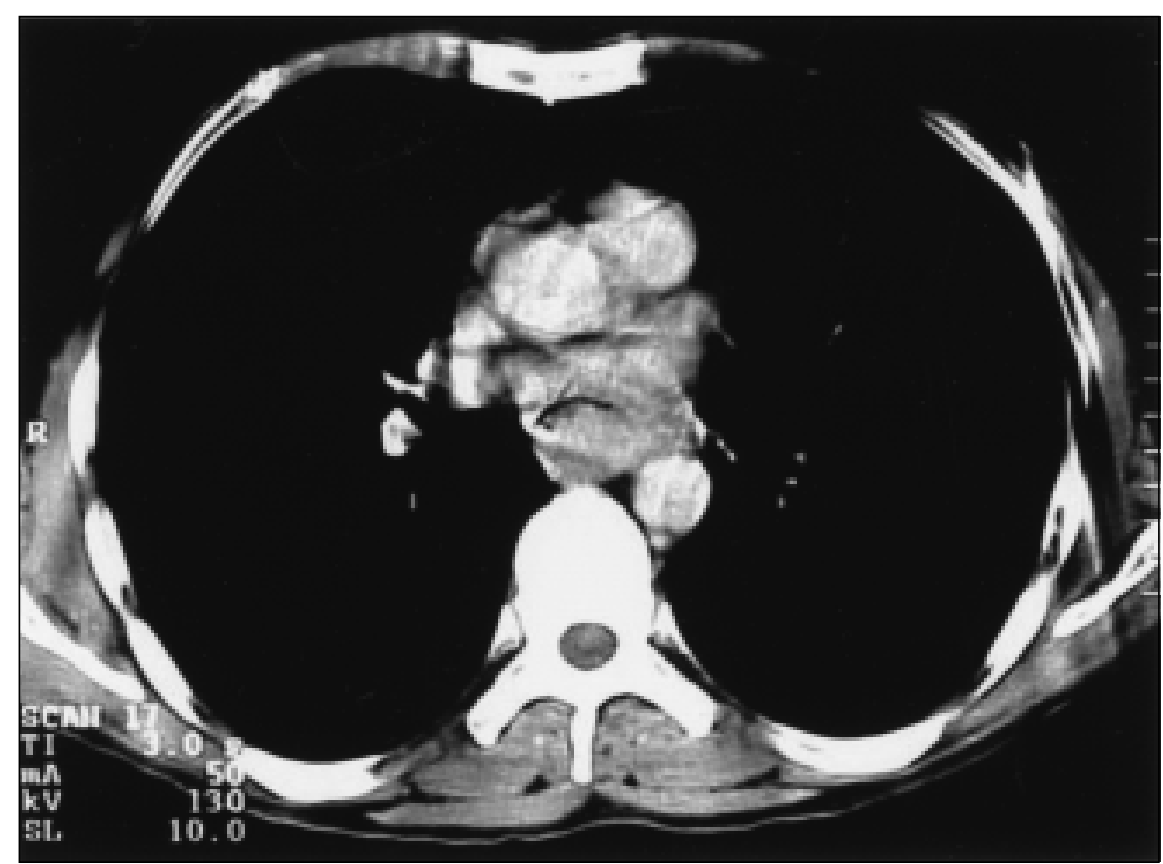

Figura 2. Tomografia computadorizada de tórax: aumento de volume e espessamento parietal no nível do terço médio do esôfago.

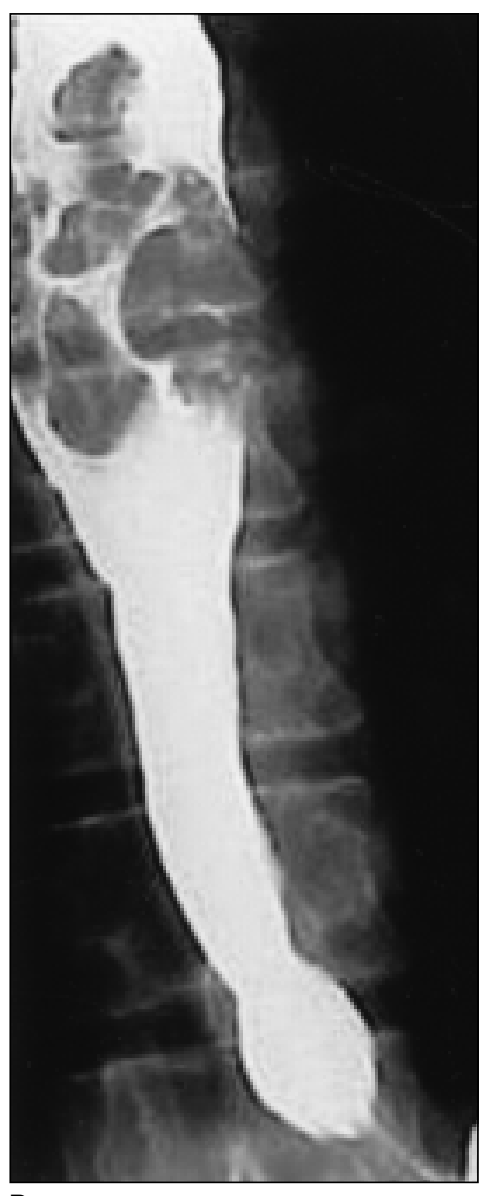

B

sarcoma, carcinoma polipóide, tumor polipóide e CEC variante de células fusiformes. Entretanto, muitos pesquisadores acreditam que essas lesões representam várias expressões de um único tumor maligno, que tem sido denominado de $\mathrm{CCF}$ ("spindle cell carcinoma")(1,2,9,10).

No passado acreditava-se que os carcinossarcomas e pseudo-sarcomas eram duas entidades distintas. O termo carcinossarcoma era utilizado para os tumores que continham uma verdadeira mistura de elementos carcinomatosos e sarcomatosos, e que ambos os componentes poderiam fornecer metástases para linfonodos regionais ou estruturas distantes ${ }^{(\mathbf{1 , 1 1}, \mathbf{1 2})}$. Por outro lado, os pseudo-sarcomas eram vistos como tumores compostos essencialmente por células fusiformes "sarcoma-like" com áreas adjacentes de CEC, dispostos lado a lado, sem haver a mistura das células. Acreditava-se que as porções sarcomatosas desse tumor não forneciam metástases para outras estruturas, e que eram lesões menos agressivas e tinham melhor prognóstico que os carcinossarcomas ${ }^{(11-15)}$. Entretanto, em estudos subseqüentes, foi demonstrado que metástases locais ou a distância ocorreram de porções sarcomatosas dos chamados pseudo-sarcomas, e essas lesões se comportaram tão agressivamente quanto os carcinossarcomas ${ }^{(\mathbf{1 6}, 17)}$. Então, carcinossarcomas e pseudo-sarcomas parecem ser a mesma entidade patológica, com graus variáveis de metaplasia de células fusiformes anaplásicas das porções carcinomatosas do tumor ${ }^{(\mathbf{1 , 9 , 1 8 , 1 9 )}}$

O CCF é um tumor que acomete principalmente homens $(5: 1)$, na faixa etária da quinta ou sexta décadas, com história prévia de tabagismo ou alcoolismo. Os sintomas clínicos são disfagia progressiva, emagrecimento e menos comumente dor retroesternal $^{(\mathbf{1}, \mathbf{4}, \mathbf{6})}$.

Ao estudo radiográfico com administração de contraste baritado esses tumores apresentam-se como grande massa polipóide alongada localizada no terço médio do esôfago, com expansão local de sua luz. $\mathrm{O}$ contorno pode ser lobulado ou ondulado, algumas vezes podendo criar um "efeito cúpula" na porção intraluminal do tumor. Pode haver um pedículo e ulcerações de mucosa de vários tamanhos. Embora a massa seja volumosa, existe relativamente 


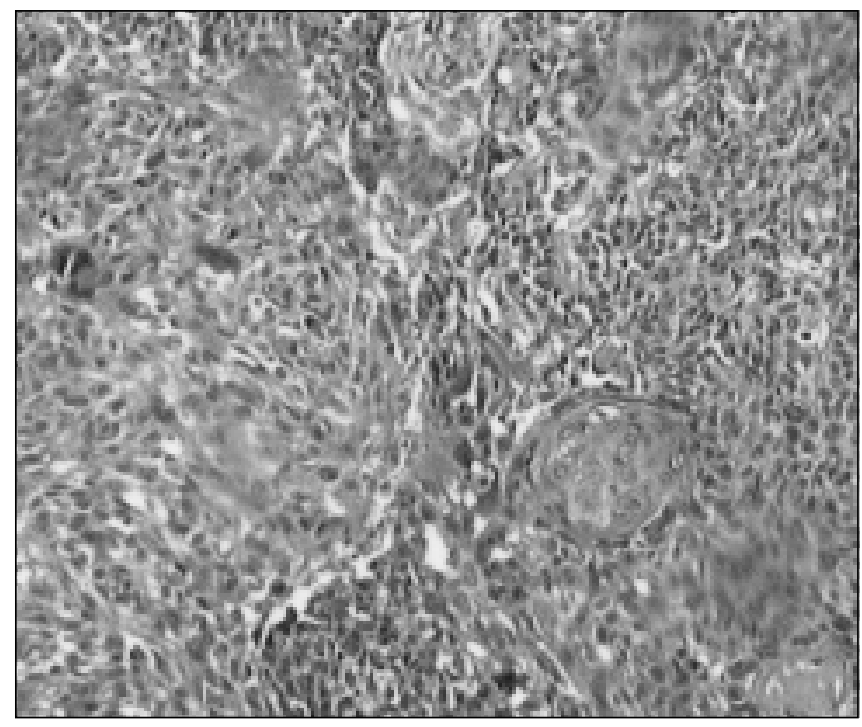

A

Figura 3. Fotomicrografias mostram duplo componente da neoplasia, com blocos sólidos de células arredondadas, com pontes intercelulares e áreas de queratinização concêntrica (pérolas córneas), e áreas fusocelulares de aspecto sarcomatóide, com intensas atipias nucleares. A: HE, 100×. B: HE, 200×.

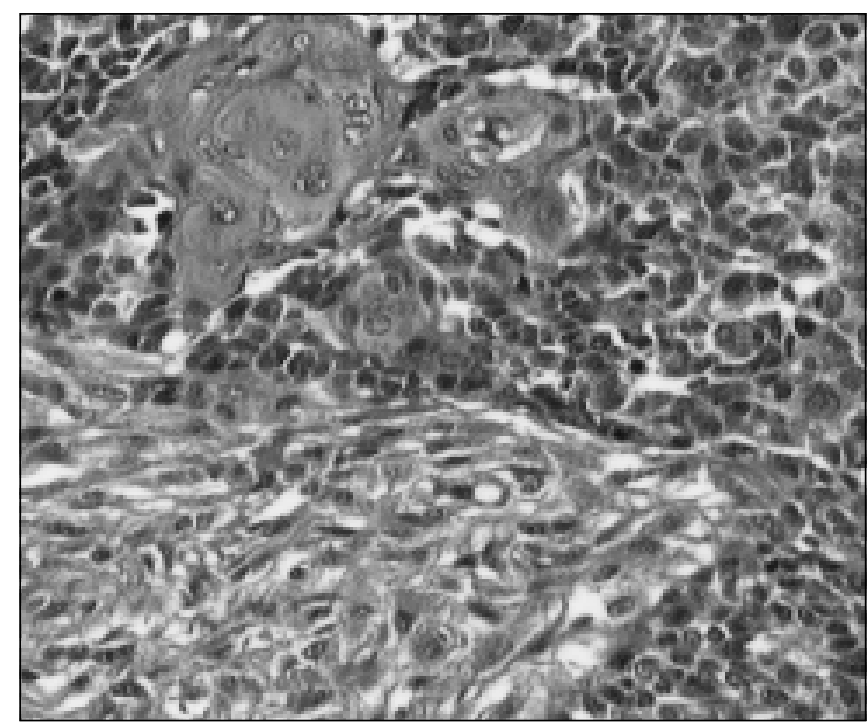

B pequena obstrução à passagem do meio de contraste $^{(\mathbf{1 , 4 , 1 0 , 1 6 )}}$. A tomografia computadorizada geralmente demonstra apenas volumosa massa de partes moles expandindo a luz do esôfago ${ }^{(2)}$.

Macroscopicamente, os CCFs apresentam-se como massas polipóides, geralmente aderidos à parede do esôfago por um pedículo espesso e curto e geralmente orientado longitudinalmente no seu maior eixo. Às vezes não há pedículo. Sua superfície pode ser lobulada ou lisa, mas a maioria possui pequenas erosões. A mucosa ao seu redor é grosseiramente normal e a luz do órgão geralmente está dilatada ${ }^{(3)}$.

Microscopicamente, a característica patológica do CCF é a coexistência de elementos carcinomatosos e sarcomatosos na lesão, ambos misturados juntos ou separados em localização. O elemento sarcomatoso é encontrado principalmente na porção polipóide do tumor, sendo geralmente representado por sarcoma de células indiferenciadas, fibrossarcoma, leiomiossarcoma, osteocondrossarcoma ou rabdomiossarcoma. $\mathrm{O}$ elemento carcinomatoso é encontrado principalmente na membrana mucosa ao redor do pedículo ou na superfície da massa polipóide, normalmente é representado por CEC, mas adenocarcinoma também já foi descrito ${ }^{(3,14)}$.

As metástases podem ser puramente carcinomatosas, puramente sarcomatosas ou mesmo mistas, sendo linfonodos regionais, pulmões e pleura os locais preferenciais de disseminação ${ }^{(\mathbf{1 , 1 6})}$.

É importante salientar que nos CCFs a biópsia realizada durante a endoscopia digestiva alta pode revelar a presença de apenas células epiteliais (mais comum devido à localização superficial) ou apenas células sarcomatosas, podendo levar a um diagnóstico equivocado ${ }^{(3)}$.

Nos diagnósticos diferenciais de lesões polipóides volumosas do esôfago incluemse outras entidades benignas e malignas. Entre as benignas estão lesões incomuns como o pólipo fibrovascular, o miofibroma, o lipoma pedunculado e o liomioma, mas geralmente essas lesões tendem a apresentar um contorno mais liso e menos lobulado $^{(\mathbf{2 0})}$. Entre as entidades malignas, os CECs e adenocarcinomas originados da mucosa de Barrett podem ser polipóides, mas essas lesões usualmente já estarão em estágio mais avançado, tendendo a infiltrar e estreitar a luz do esôfago, e não a expandi-la ${ }^{(2,5)}$. Outros tumores raros do esôfago, tais como liomiossarcoma, fibrossarcoma, rabdomiossarcoma, melanoma, carcinoma de células pequenas, linfoma e sarcoma de Kaposi também podem se manifestar como lesões polipóides intraluminais ${ }^{(\mathbf{1 , 2})}$. Desse modo, o diagnóstico definitivo de CCF só pode ser realizado por intermédio de estudo histopatológico.
$\mathrm{O}$ tratamento recomendado para o $\mathrm{CCF}$ de esôfago é a esofagectomia radical, porém a terapia ideal ainda continua em investigação $^{(4)}$.

Foi previamente sugerido que os CCFs teriam um prognóstico melhor do que os CECs, devido à sua localização superficial, com invasão local e regional ou metástases a distância ocorrendo tardiamente no curso da doença ${ }^{(16,17)}$. Entretanto, outros investigadores encontraram que cerca de $50 \%$ dos pacientes com CCF de esôfago têm doença metastática no momento do diagnóstico, e a sobrevida média em cinco anos é de apenas $2 \%$ a $8 \%{ }^{(2,12,21)}$. Então, o prognóstico deste tumor é provavelmente comparável ao do CEC.

\section{REFERÊNCIAS}

1. Agha FP, Keren DF. Spindle-cell squamous carcinoma of the esophagus: a tumor with biphasic morphology. AJR 1985;145:541-5.

2. Levine MS. Other malignant tumors of the esophagus. In: Gore RM, ed. Textbook of gastrointestinal radiology. 2nd ed. Philadelphia: WB Saunders, 1994:435-51.

3. Xu LT, Sun CF, Wu LH, Chang ZR, Liu TH. Clinical and pathological characteristics of carcinosarcoma of the esophagus: report of four cases. Ann Thorac Surg 1984;37:197-203.

4. Hinderleider CD, Aguam AS, Wilder JR. Carcinosarcoma of the esophagus: a case report and review of the literature. Int Surg 1979;64:13-9.

5. Levine MS. Esophageal cancer. Radiologic diagnosis. Radiol Clin North Am 1997;35:265-79.

6. Nichols T, Yokoo H, Craig RM, Shields TW. Pseudosarcoma of the esophagus. Three new cases and 
review of the literature. Am J Gastroenterol 1979; 72:615-22.

7. Virchow RLK. Vorlesungen uber Pathologie die krankhaften Geschwulste. Berlin: A Hirschwald, 1865.

8. Von Hansemann D. Das gleichzeitge Vorkommen verschiedenartiger Geschwülste bei der selben Person. Z Krebsforsch 1904:183-98.

9. Martin MR, Kahn LB. So-called pseudosarcoma of the esophagus: nodal metastases of the spindle cell element. Arch Pathol Lab Med 1977;101:604-9.

10. Olmsted WW, Lichtenstein JE, Hyams VJ. Polypoid epithelial malignancies of the esophagus. AJR 1983;140:921-5.

11. DeMarco AR, Leon W, Coleman WO, Welsh RA, Strug LH. Pseudosarcoma of the esophagus. J Thorac Cardiovasc Surg 1965;49:188.
12. McCort JJ. Esophageal carcinosarcoma and pseudosarcoma. Radiology 1972;102:519-24.

13. Postlethwait RW, Wechsler AS, Shelburne JD. Pseudosarcoma of the esophagus. Ann Thorac Surg 1975; 19:198-205.

14. Miranda FJ, Kallas Neto JA, Andrade da Costa E, Badan Palhares FA. Carcinosarcoma of the esophagus. Int Surg 1980;65:463-7.

15. Razzuk MA, Urschel HC Jr, Race GJ, Nathan MJ, Paulson DL. Pseudosarcoma of the esophagus. A case report. J Thorac Cardiovasc Surg 1971;61: 650-3.

16. Kenneweg DJ, Cimmino CV. Carcinosarcoma of the esophagus. Am J Roentgenol Radium Ther Nucl Med 1967;101:482-4.

17. Talbert JL, Cantrell JR. Clinical and pathological characteristics of carcinosarcoma of the esophagus.
J Thorac Cardiovasc Sug 1963;45:1-12.

18. Osamura RY, Shimamura K, Hata J, et al. Polypoid carcinoma of the esophagus. A unifying term for "carcinosarcoma" and "pseudosarcoma". Am J Surg Pathol 1978;2:201-8.

19. Takubo K, Tsuchiya S, Nakagawa H, Futatsuki K, Ishibashi I, Hirata F. Pseudosarcoma of the esophagus. Hum Pathol 1982;13:503-5.

20. Levine MS, Buck JL, Pantongrag-Brown L, Buetow PC, Hallman JR, Sobin LH. Fibrovascular polyps of the esophagus: clinical, radiographic, and pathologic findings in 16 patients. AJR 1996;166:7817.

21. Dillon ML Jr, Mobin-Uddin K, Utley JR, Bryanl LR What is rational treatment for carcinoma of the esophagus and cardia? J Thorac Cardiovasc Surg 1974;68:321-7. 